Check for updates

Cite this: RSC Adv., 2019, 9, 10948

\title{
Elastic methyltrimethoxysilane based silica aerogels reinforced with polyvinylmethyldimethoxysilane
}

\begin{abstract}
Lukai Wang, Junzong Feng, Yonggang Jiang, Liangjun Li and Jian Feng (DD*
Native silica aerogels are fragile and brittle, which prevents their wider utility. For designing more durable and stronger silica aerogels, polyvinylmethyldimethoxysilane (PVMDMS) polymers as effective and multifunctional reinforcing agents were used to strengthen methyltrimethoxysilane based silica aerogels (MSAs). The PVMDMS polymer, which was composed of long-chain aliphatic hydrocarbons and organic side-chain methyl and alkoxysilane groups, was integrated into silica networks via a simple sol-gel process. Compared with MSAs, PVMDMS reinforced MSAs (PRMSAs) display many fascinating characteristics. PRMSAs exhibit improved hydrophobic properties (water contact angle of $136.9^{\circ}$ ) due to abundant methyl groups in the silica networks. Benefiting from the fine integration of PVMDMS polymers into MSAs, PRMSAs show a perfectly elastic recovery property, the compressive strength of which ranges from 0.19 to $1.98 \mathrm{MPa}$. More importantly, PVMDMS polymers have successfully suppressed the growth of secondary particles. Homogeneous silica networks formed by nanoscale particles give PRMSAs a high surface area of $1039 \mathrm{~m}^{2} \mathrm{~g}^{-1}$. Moreover, optimized PRMSAs also exhibit a low thermal conductivity of $0.0228 \mathrm{~W} \mathrm{~m}^{-1} \mathrm{~K}^{-1}$ under ambient conditions, and their thermal stability reaches up to $222.3^{\circ} \mathrm{C}$ in air. All the results obtained from this paper will help us to design silica aerogels.
\end{abstract}

Received 5th February 2019

Accepted 2nd April 2019

DOI: 10.1039/c9ra00970a

rsc.li/rsc-advances
Though the MSAs are very flexible and elastic, it does not take much force to compress them. ${ }^{11}$ For designing more durable and stronger silica aerogels, the choice of reinforcement methods, on the basis of reasonable selection of silane precursors, is also a crucial step. Up to now, different approaches have been explored to reinforce silica aerogels, which include enhancing gel strength by increasing gel aging temperature, ${ }^{12}$ reinforcing the crosslink of silica backbone with polymers, ${ }^{4,13,14}$ and dispersing different fibers in the initial sol of silica aerogels, ${ }^{15,16}$ etc. In principle, the most effective way is the integration of polymers into silica aerogels. Thus, the organicinorganic hybrid silica aerogels prepared by this method have more robust structures and better mechanical properties. ${ }^{17}$

At present, these polymers mainly include epoxide, ${ }^{18}$ polyurea,${ }^{19}$ polyurethane,${ }^{20}$ polyacrylonitrile, ${ }^{21}$ and polystyrene, ${ }^{22}$ etc. And their reinforcement mechanism can be achieved by, firstly modifying the surface chemistry of silica networks with appropriate functional groups and then growing the polymer coatings from modified surfaces. ${ }^{17}$ Thus, aerogels reinforced by these polymers usually exhibit heterogeneous core-shell structures, ${ }^{\mathbf{1}}$ which would be unfavorable to satisfy the requirements of silica aerogels with light weight, high surface area, and excellent insulation performance. Recently, Kanamori's group ${ }^{23-25}$ has reported an innovative approach to prepare doubly cross-linked polyvinylpolydimethylsiloxane-based aerogels. This novel and facile preparation of the double-cross-linking structure is based on consecutive processes involving radical polymerization and hydrolytic polycondensation. ${ }^{25}$ In contrast to core-shell
Science and Technology on Advanced Ceramic Fibers and Composites Laboratory, College of Aerospace Science and Engineering, National University of Defense Technology, 109 De Ya Rd, Changsha, Hunan, 410073, P. R. China. E-mail: fengj@ nudt.edu.cn 
structures, the doubly cross-linked strategy is more conducive to forming homogeneous silica networks, avoiding obvious phase separation ${ }^{23}$ and particles aggregation in the sol-gel process. Benefiting from the organic bridge and homogeneous distribution of polyvinylmethyldimethoxysilane (PVMDMS) in silica networks, polyvinylpolydimethylsiloxane aerogels exhibit many merits, such as low density, uniform nanopores, high transparency, super compressibility, high bendability, excellent machinability, and thermal superinsulation. ${ }^{24,25}$ However, the plastic deformation still exists in polyvinylpolydimethylsiloxane aerogel networks to some extent. These aerogels obtained by supercritical drying cannot rebound to their original height when the compressive stress is removed.

Inspired by the double cross-linking strategy, polyorganoalkoxysilane with high molecular weight is a kind of ideal polymer reinforcing agents for silica aerogels. Herein, we report the design of an elastic polymer-reinforced silica aerogel, PVMDMS reinforced MTMS based silica aerogel (PRMSA), which was facilely prepared by compounding PVMDMS polymers into the initial sol of MSAs. An extremely fascinating phenomenon was discovered that PRMSAs with abundant methyl groups and long-chain aliphatic hydrocarbons exhibit wonderfully elastic recovery property. In this paper, the hydrophobicity, morphology, specific surface area (SSA), and mechanical properties of PRMSAs were characterized systematically. Furthermore, the thermal conductivity and thermostability were also investigated. Excellent thermal insulation properties would ensure that PRMSAs are more suitable for energy-saving applications, although organic components may have some negative effects on the thermal stability.

\section{Experimental section}

\subsection{Materials}

Vinylmethyldimethoxysilane (purity: 97\%), di-tert-butyl peroxide (purity: 97\%), methyltrimethoxysilane (purity: 97\%), tetramethylammonium hydroxide pentahydrate (purity: 97\%), benzyl alcohol (GC), ethanol (AR), and distilled water were purchased from Shanghai Aladdin Biochemical Technology Co., Ltd. All reagents in this work were used as received without further purification.

\subsection{Preparation of PVMDMS}

According to Zu's method, ${ }^{23-25}$ we have successfully prepared PVMDMS polymers. The mixture of vinylmethyldimethoxysilane (VMDMS) and di-tert-butyl peroxide (DTBP) with a molar ratio of 10 : 1 was added to the hydrothermal reactor, and argon was injected into the hydrothermal reactor three times to remove air. Then, the hydrothermal reactor was sealed and heated at $120^{\circ} \mathrm{C}$ for $72 \mathrm{~h}$. After reaction, the transparent PVMDMS can be obtained by cooling to room temperature. The weight-average molecular weight of these polymers can reach up to 13982 , and the degree of polymerization was up to 105 .

\subsection{Preparation of PRMSAs}

Fig. 1 displays the synthesis process of PRMSAs. For the sol-gel stage, firstly, PVMDMS and MTMS were mixed evenly in benzyl alcohol (BzOH) solvent. The Si molar percentage of PVMDMS/ (PVMDMS + MTMS) was controlled at 20-90\% $\mathrm{mol} \mathrm{mol}^{-1}$. And the molar ratio of precursors to $\mathrm{BzOH}$ was controlled at $\mathrm{BzOH} / \mathrm{Si}=5 \mathrm{~mol} \mathrm{~mol}^{-1}$. The uniformly dispersed transparent

(a) Molecular structure of PRMSAs

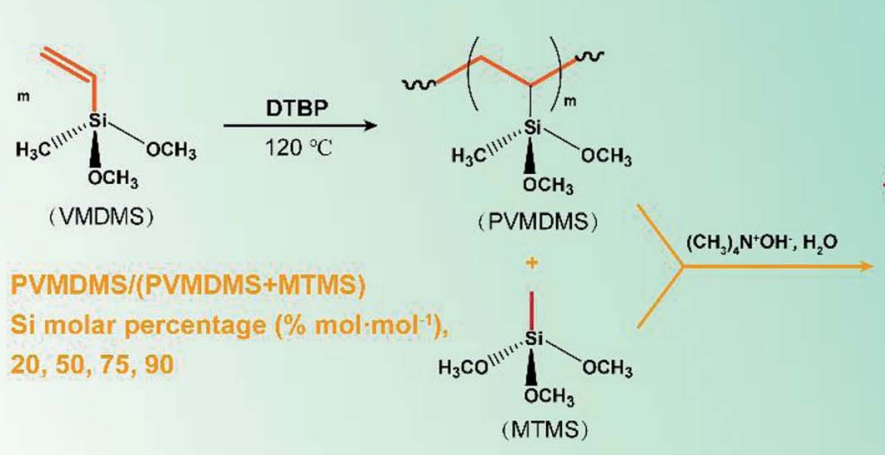

(b) Synthesis process of PRMSAs

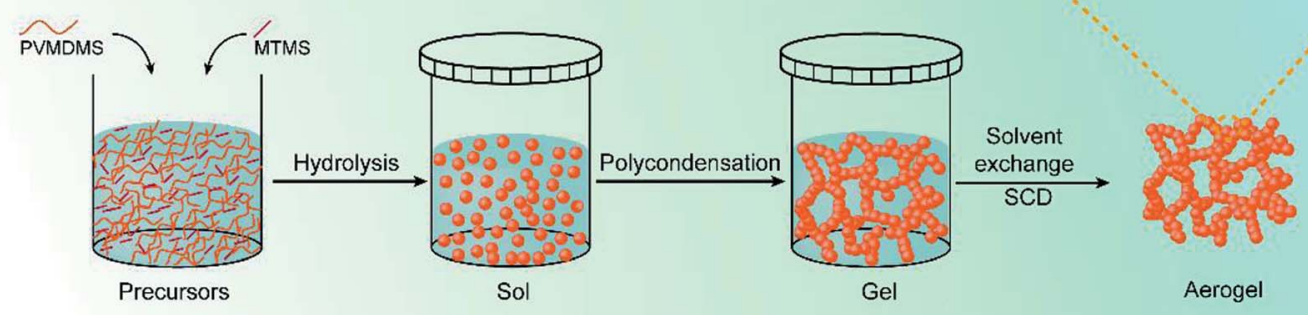

Fig. 1 Schematic diagram of preparation process of PRMSAs. (a) Molecular structure of PRMSAs; (b) synthesis process of PRMSAs. 
solution was obtained by slowly stirring. Then, tetramethylammonium hydroxide (TMAOH) $/ \mathrm{Si}=0.075 \mathrm{~mol} \mathrm{~mol}^{-1}$ and deionized water $\left(\mathrm{H}_{2} \mathrm{O}\right) / \mathrm{Si}=3 \mathrm{~mol} \mathrm{~mol}^{-1}$ were added into the transparent solution, and the homogeneous sol was obtained by slowly stirring for $10 \mathrm{~min}$. After that, the sol was transported into a plastic container with a lid and sealed. The gel was obtained at $50{ }^{\circ} \mathrm{C}$ for $2 \mathrm{~h}$ and aged at $100{ }^{\circ} \mathrm{C}$ for $4 \mathrm{~d}$ in an oven, and after 4 times of ethanol solvent exchange (each $24 \mathrm{~h}$ ) at $60^{\circ} \mathrm{C}$ to wash and remove the residuals, the orange gel turned to a transparent gel. Finally, PRMSAs were obtained after experiencing $\mathrm{CO}_{2}$ supercritical drying $\left(55^{\circ} \mathrm{C}, 15 \mathrm{MPa}\right)$.

\subsection{Characterizations}

The bulk density $\left(\rho_{\mathrm{b}}\right)$ was determined by measuring the weight and volume of samples. The diameter shrinkage and volume shrinkage were measured by the ratio of size change from wet gels to aerogels. The weight-average molecular weight and polydispersity of PVMDMS were determined by gel permeation chromatography (GPC) system (PL-GPC50, Agilent, Germany) with chloroform as solvent. The hydrophobic performance of specimens was measured by using contact angle instrument (DSA100, KRUSS, Germany) with putting a $5 \mu \mathrm{L}$ water drop on the horizontal surface of aerogels. Fourier-transform infrared (FTIR) spectra of all samples were measured at $400-4000 \mathrm{~cm}^{-1}$ using pressed $\mathrm{KBr}$ pellets on a spectrophotometer (Nicolet 6700, Thermo fisher, USA; resolution: $2 \mathrm{~cm}^{-1}$ ). Uniaxial compressiondecompression tests were carried out with a material tester (XBD-4000, Xinbiao Corp., China). The crosshead speed was 0.5 $\mathrm{mm} \mathrm{min}^{-1}$ for compression and $0.2 \mathrm{~mm} \mathrm{~min}^{-1}$ for decompression. The surface structure and morphology of specimens were observed by using a field-emission scanning electron microscope (FESEM; SIGMA 500, ZEISS, Germany) operated at $10 \mathrm{kV}$. The samples were sputter covered with a gold alloy prior to imaging. Nitrogen adsorption-desorption isotherms were calculated at $77 \mathrm{~K}$ through a Quantachrome ASiQwin surface area and porosimetry analyzer. Aerogel powders were degassed under vacuum at $80^{\circ} \mathrm{C}$ for $8 \mathrm{~h}$ prior to the analysis. The SSA of aerogels was determined from the adsorption isotherm using the Brunauer-Emmett-Teller (BET) theory through an automatic surface area analyzer (Autosorb-iQ $\mathrm{i}_{2} \mathrm{MP}$, Quantachrome, USA) at $\mathrm{N}_{2}$ environment. The pore size distribution was derived from desorption branches of the isotherms by applying the Barrett-Joyner-Halenda (BJH) method. According to open literature ${ }^{26}$ the average pore size was calculated by eqn (1), and the pore volume $\left(V_{\text {pore }}\right)$ was obtained by eqn (2). The skeleton density $\left(\rho_{\mathrm{s}}\right)$ of silica aerogels is $2.2 \mathrm{~g} \mathrm{~cm}^{-3}$, which was obtained from reported work. ${ }^{27}$ The thermal conductivity of aerogels was tested by using a Hot Disk thermal constant analyzer (TPS2500S, Hot Disk, Sweden) with the c5465 detector. And the thermal conductivity of PRMSAs with the size of $\varnothing 30 \times 20 \mathrm{~mm}$ was measured under "bulk" mode. Each sample was tested for 3 times and the average value was adopted. To evaluate the thermal stability of samples, TG-DSC analyses were performed on a thermogravimetric Analyzer (STA 449 F3, NETZSCH, Germany) in air atmosphere, with a heating rate of $10{ }^{\circ} \mathrm{C} \mathrm{min}^{-1}$ from room temperature to $800{ }^{\circ} \mathrm{C}$.

$$
\begin{gathered}
\text { Average pore size }=\frac{4 V_{\text {pore }}}{S_{\mathrm{BET}}} \\
V_{\text {pore }}=\frac{1}{\rho_{\mathrm{b}}}-\frac{1}{\rho_{\mathrm{s}}}
\end{gathered}
$$

\section{Results and discussion}

\subsection{Hydrophobicity of monolithic PRMSAs}

The reasonable selection of silane precursors is a crucial step for synthesizing silica aerogels. In order to prepare mechanically enhanced silica aerogels, our group has chosen hydrophobic MTMS as the silane precursor and PVMDMS polymer with methyl groups as the reinforcement to overcome the brittleness issue of native silica aerogels. The wet gel was formed by strong base catalysis via the sol-gel process, and the resultant PRMSAs were prepared by exchanging solvent inside gels with supercritical fluids. The detailed preparation process is shown in Fig. 1. Compare with MSAs in Fig. 2a, PRMSAs exhibit better monolithic properties without significant cracking and fragmentation. The reason for forming monolithic aerogels should be explained from the viewpoint of molecular structure. The successful integration of polyorganoalkoxysilane into inorganic silica system makes silica aerogels possess long-chain aliphatic hydrocarbons and abundant methyl groups, which plays a vital role in improving the mechanical property of silica aerogels.

Apart from mechanical properties, hydrophobicity is also worth mentioning. The hydrophobic nature is quite important for silica aerogels to avoid their deterioration with environmental humidity and to maintain their cleanliness. Thus, the reasonable design of hydrophobicity would be beneficial to the widely practical application of silica aerogels. Table 1 displays the hydrophobicity characterized by measuring the contact angle of water. Respectively, the contact angles of PRMSA-50 and MSA are $136^{\circ}$ and $120^{\circ}$ in Fig. $3 \mathrm{~b}$ and d. The high contact angle of PRMSA-50 may be attributed to its hydrophobic aliphatic hydrocarbons. To further verify the hydrophobicity, PRMSA-50 shown in Fig. 2c was placed in water. After a long time of immersion, the aerogel could still float on the water surface without obvious water absorption. PRMSA-20, PRMSA50, PRMSA-75, and PRMSA-90, which are reinforced with different dosages of PVMDMS, show different contact angles. PRMSA-20, PRMSA-75, and PRMSA-90 exhibit relatively low contact angles compared to PRMSA-50 in Table 1. The unsatisfied hydrophobic performance of PRMSA-20 may be caused by the low content of PVMDMS. Differently, the low hydrophobicity of PRMSA-75 and PRMSA-90 could be account for their relatively dense structure (Fig. $4 \mathrm{~g}$ and $\mathrm{h}$ ). In fact, these results mean that the proper introduction of PVMDMS could lead to an improved hydrophobicity.

The five presented spectra in Fig. 3 suggest a chemical structure with similar silica backbone, showing the existence of an organically modified silica network. Indeed, the absorption peaks at 1140,1038 , and $780 \mathrm{~cm}^{-1}$ are ascribed to the stretching vibrations of $\mathrm{Si}-\mathrm{O}-\mathrm{Si}$ bonds, and the peak at $440 \mathrm{~cm}^{-1}$ 

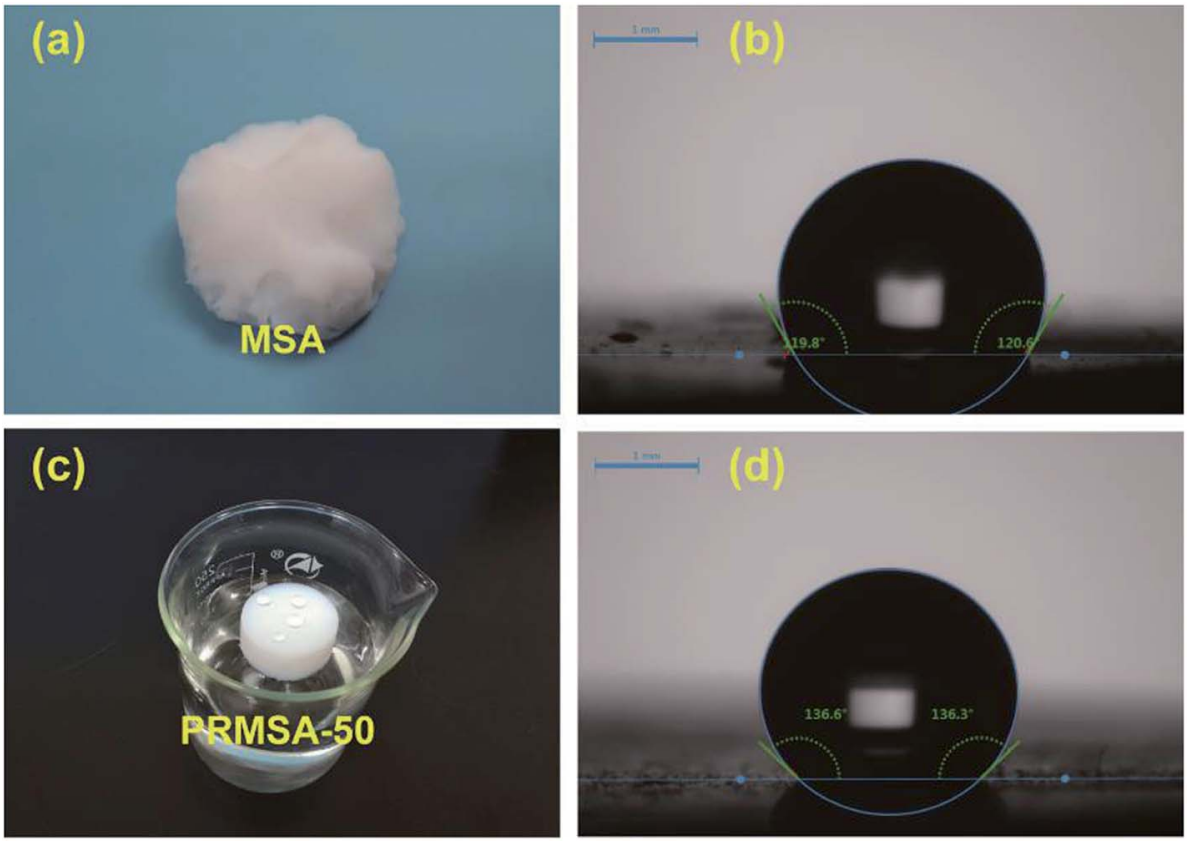

Fig. 2 Hydrophobic performances of MSAs and PRMSAs. (a) MSA; (b) water contact angle of MSA; (c) PRMSA-50; (d) water contact angle of PRMSA-50.

Table 1 Physical properties of MSAs and PRMSAs

\begin{tabular}{llccr}
\hline Sample & Density $\left(\mathrm{g} \mathrm{cm}^{-3}\right)$ & Diameter shrinkage $(\%)$ & Volume shrinkage $(\%)$ & Contact angle of water $\left(^{\circ}\right)$ \\
\hline MSA & - & - & - & $119.8 \pm 0.6$ \\
PRMSA-20 & $0.110 \pm 0.002$ & $4.77 \pm 0.17$ & $13.00 \pm 0.26$ & $130.2 \pm 0.4$ \\
PRMSA-50 & $0.138 \pm 0.002$ & $7.88 \pm 0.10$ & $18.17 \pm 0.17$ & $136.9 \pm 0.7$ \\
PRMSA-75 & $0.165 \pm 0.001$ & $10.45 \pm 0.43$ & $22.11 \pm 0.63$ & $133.2 \pm 0.4$ \\
PRMSA-90 & $0.170 \pm 0.001$ & $10.89 \pm 0.27$ & $24.55 \pm 0.39$ & $131.8 \pm 0.8$
\end{tabular}

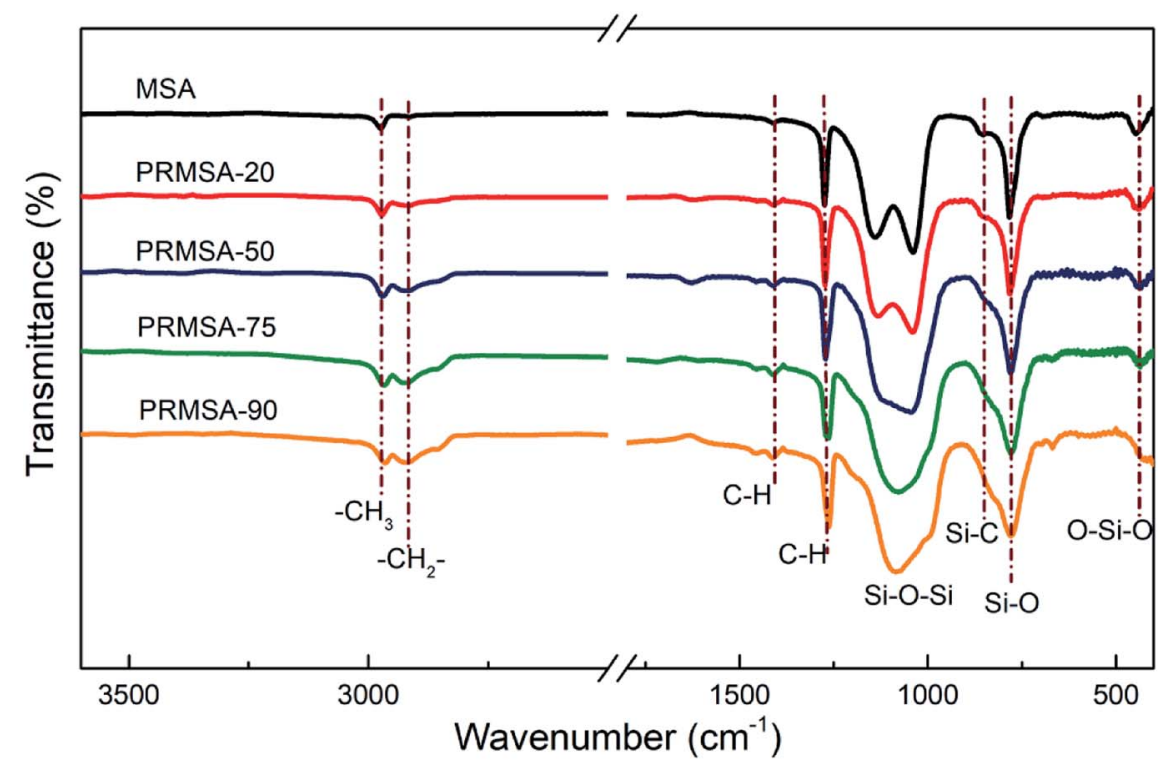

Fig. 3 FTIR spectra of MSAs and PRMSAs. 

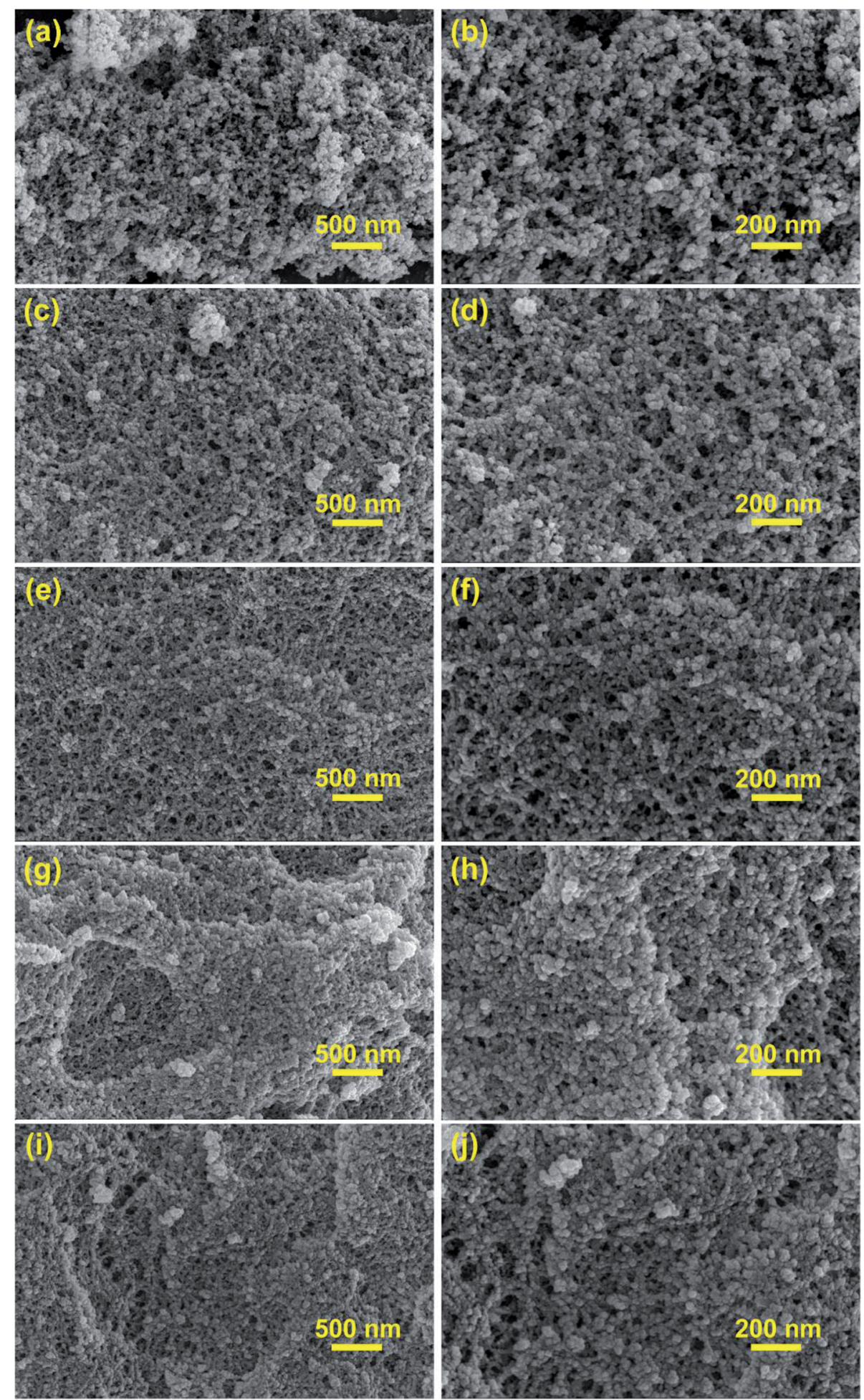

Fig. 4 SEM images of aerogels synthesized by varying compositions. (a and b) MSA; (c and d) PRMSA-20; (e and f) PRMSA-50; (g and h) PRMSA75; (i and j) PRMSA-90.

corresponds to the bending vibration of O-Si-O bonds. ${ }^{28}$ Abundant methyl groups are the essential factor to prepare hydrophobic silica aerogels. The presence of methyl groups is proven by the FTIR spectra, which originates stretching vibrations of the $\mathrm{C}-\mathrm{H}$ bonds that are found at $2972 \mathrm{~cm}^{-1}$, and the stretching vibrations of $\mathrm{Si}-\mathrm{C}$ bonds near $850 \mathrm{~cm}^{-1}$. The stretching vibrations of methylene groups are also observed at $2925 \mathrm{~cm}^{-1}$, and the vibration absorption peaks are obvious with the increase of PVMDMS dosage. Respectively, the abovedescribed peaks are coincident with those already reported in open literature, ${ }^{29}$ which also means that PVMDMS reinforcements are successfully integrated into silica aerogels. 


\subsection{Morphology and porous structure}

The morphological structures of MSAs and PRMSAs have been investigated by SEM images in Fig. 4. The similarity of these aerogels is that their skeleton networks consist of a large number of nanoparticles, which would be beneficial to acquire a uniform silica network. But for MSA, there are also some relatively large secondary particles $(\sim 500 \mathrm{~nm})$ formed by aggregating small nanoparticles (Fig. 4a and b), which are responsible for the existence of large pores in MSA. More interestingly, the growth of secondary particles could be inhibited with the increased dosage of PVMDMS. As shown in SEM images (Fig. 4c-j), the obtained PRMSA-20, PRMSA-50, PRMSA-75, and PRMSA-90 exhibit a relatively homogeneous three-dimensional porous network structure. The pore size of PRMSAs is a bit smaller than that of MSA, meaning that continuous addition of polymers could avoid the collapse of aerogel networks, and inhibit the damage of capillary tension. However, it is noteworthy that excessive dosage of PVMDMS reinforcements may cause a more dense structure and higher density due to the polymer shrinkage, which would have a negative impact on the thermal insulation and high hydrophobic properties of PRMSAs.

Aerogels are a kind of typical porous materials, which have attracted wide attention due to their attractive surface area and porous structure. In our experiments, the SSA of aerogels measured by the BET method has been shown in Table 2. The SSA of MSA is up to $544 \mathrm{~m}^{2} \mathrm{~g}^{-1}$. Compared with MSA, PRMSAs exhibit a relatively large SSA owing to these uniform nanopores (Fig. 5b) in doubly cross-linked silica networks. Respectively, the SSA of PRMSA-75 is twice as high as that of MSA, reaching up to $1039 \mathrm{~m}^{2} \mathrm{~g}^{-1}$. Moreover, the SSA of PRMSAs is also closely related with aerogel network structures. Because of the fine solution of PVMDMS polymers in the original sol, there is no obvious phase separation in the sol-gel process. PRMSA-20, PRMSA-50, PRMSA-75, and PRMSA-90 with homogeneous network structure exhibit a higher SSA than that of MSA with large secondary particles in its network. Generally, these obtained results would be meaningful for synthesizing hybrid silica aerogels with high SSA.

Fig. 5a shows the nitrogen adsorption-desorption isotherms of aerogels. All aerogels exhibit lower adsorption at the relative pressure range of $0-0.7$, and adsorption monotonically increased with increasing relative pressure, which is characteristic of type IV isotherms. ${ }^{30}$ PRMSA-20, PRMSA-50, PRMSA-75, and PRMSA-90 show similar nitrogen adsorption-desorption isotherms in Fig. 5a. The hysteresis loops of PRMSAs are larger than that of MSA, which indicates the existence of a substantial amount of pores in PRMSA networks. Fig. 5b shows the pore size distribution of aerogels. The MSA shows a peak of pore size distribution at $\sim 21 \mathrm{~nm}$. Compared with MSA, PRMSAs have high peaks of pore size distribution at the range from 11 to $14 \mathrm{~nm}$. These more uniform and smaller nanopores also indicate that PVMDMS reinforcements are helpful for forming homogenous silica networks. Meanwhile, the calculated pore volume ranges from 5.428 to $8.636 \mathrm{~cm}^{3} \mathrm{~g}^{-1}$ in Table 2 , which indirectly proves that PVMDMS plays an important role in controlling the porous structure of aerogels.

Table 2 Pore structure features of MSAs and PRMSAs

\begin{tabular}{llcccc}
\hline Sample & Si molar percentage & \\
\hline MSA & 0 & $S_{\mathrm{BET}}^{b}\left(\mathrm{~mol} \mathrm{~mol}^{2} \mathrm{~g}^{-1}\right)$ & $d^{c}(\mathrm{~nm})$ & $V_{\text {pore }}^{d}\left(\mathrm{~cm}^{3} \mathrm{~g}^{-1}\right)$ & Average pore size $^{e}\left(\mathrm{~nm}^{-1}\right)$ \\
PRMSA-20 & 20 & 544 & 21.16 & - & - \\
PRMSA-50 & 50 & 795 & 12.51 & 8.636 & 43.45 \\
PRMSA-75 & 75 & 987 & 11.07 & 6.791 & 27.52 \\
PRMSA-90 & 90 & 1039 & 10.97 & 5.606 & 21.58 \\
\end{tabular}

${ }^{a}$ Si molar percentage of PVMDMS/(PVMDMS + MTMS). ${ }^{b}$ SSA obtained from nitrogen adsorption isotherm. ${ }^{c}$ Mean pore diameter obtained via BJH method. ${ }^{d} V_{\text {pore }}$ acquired by eqn (2). ${ }^{e}$ Average pore size calculated by eqn (1).
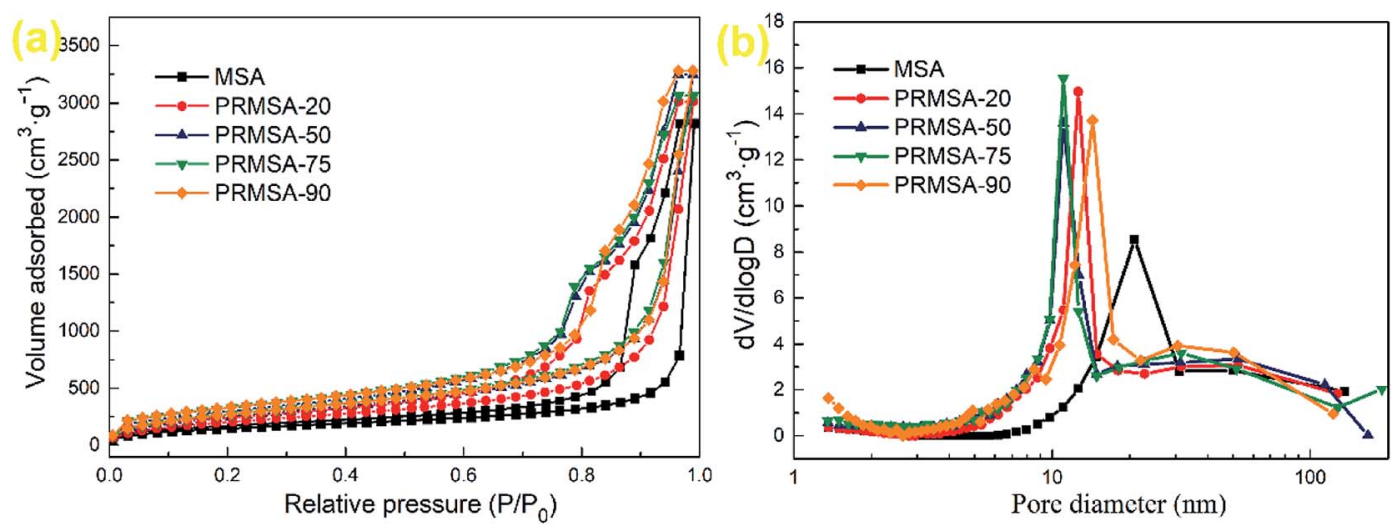

Fig. 5 Porous structures of MSAs and PRMSAs. (a) $\mathrm{N}_{2}$ adsorption-desorption isotherms; (b) pore size distributions obtained via BJH method. 


\subsection{Mechanical properties}

It has been reported that monolithic MSAs could be prepared by using MTMS as the sole precursor. ${ }^{31}$ Further developments, owing to the effect of urea and surfactant, MSAs exhibit a high compressive modulus of $7.41 \mathrm{MPa} .^{32}$ Different from previous researches, the mechanical property of MSAs has been improved by introducing PVMDMS polymers into silica networks in this paper. Fig. $6 \mathrm{a}$ and $\mathrm{b}$ show the stress-strain curves of PRMSAs with different dosages of PVMDMS. As presented in these stress-strain curves of uniaxial compressiondecompression tests, the optimized aerogels PRMSA-20 and PRMSA-50 show fine elastic recovery properties. It can be observed from Fig. $6 \mathrm{~b}$ that PRMSA-20 and PRMSA-50 compressed with $50 \%$ strain have no obvious fracture and can fully recover their original shapes after the force is removed. In addition, in contrast to compression processes in Fig. 6a and b, there is an elastic network in PRMSA-20 and PRMSA-50, which gives them a greater resilience force during decompression processes. A possible explanation for this phenomenon may be that appropriate addition of PVMDMS inhibits the inherent brittleness of silica aerogels, and greatly facilitates the formation of a flexible silica network by self-crosslinking.

The Young's moduli of PRMSAs calculated from the stressstrain curves of uniaxial compression-decompression tests have been displayed in Fig. $6 \mathrm{c}$ and d. These values of Young's moduli suggest that PRMSAs prepared with more polymers usually have higher Young's modulus. However, there is a negative correlation between the elastic behavior of aerogels and Young's modulus. For example, the Young's modulus of PRMSA-90 is higher than that of other samples at $20 \%$ strain or $50 \%$ strain, but the elastic recovery of PRMSA-90 is not satisfactory in Fig. 6a and b. In fact, PVMDMS polymers with alkoxysilane side chains have effectively enhanced the internal pearl-like structure of silica aerogels, which avoids the formation of fragile silica ceramic phase by aggregating nanoparticles. Thus, the compressive strength of PRMSAs is determined by the addition of PVMDMS polymers, which is consistent with Young's modulus in Fig. 6c and d. Moreover, it is noteworthy that the compressive strength of PRMSAs prepared by one pot catalysis with a strong base is significantly higher than that of other polymer modified silica aerogels. ${ }^{33-35}$ After the compression tests, PRMSAs can maintain a fine monolithic shape without cracking and fragmentation, which would be of great significance for the practical application of silica aerogels.

\subsection{Thermal properties}

In the field of thermal insulation, thermal conductivity is an important index for evaluating the quality of PRMSAs. The thermal conductivity of a porous material consists of three
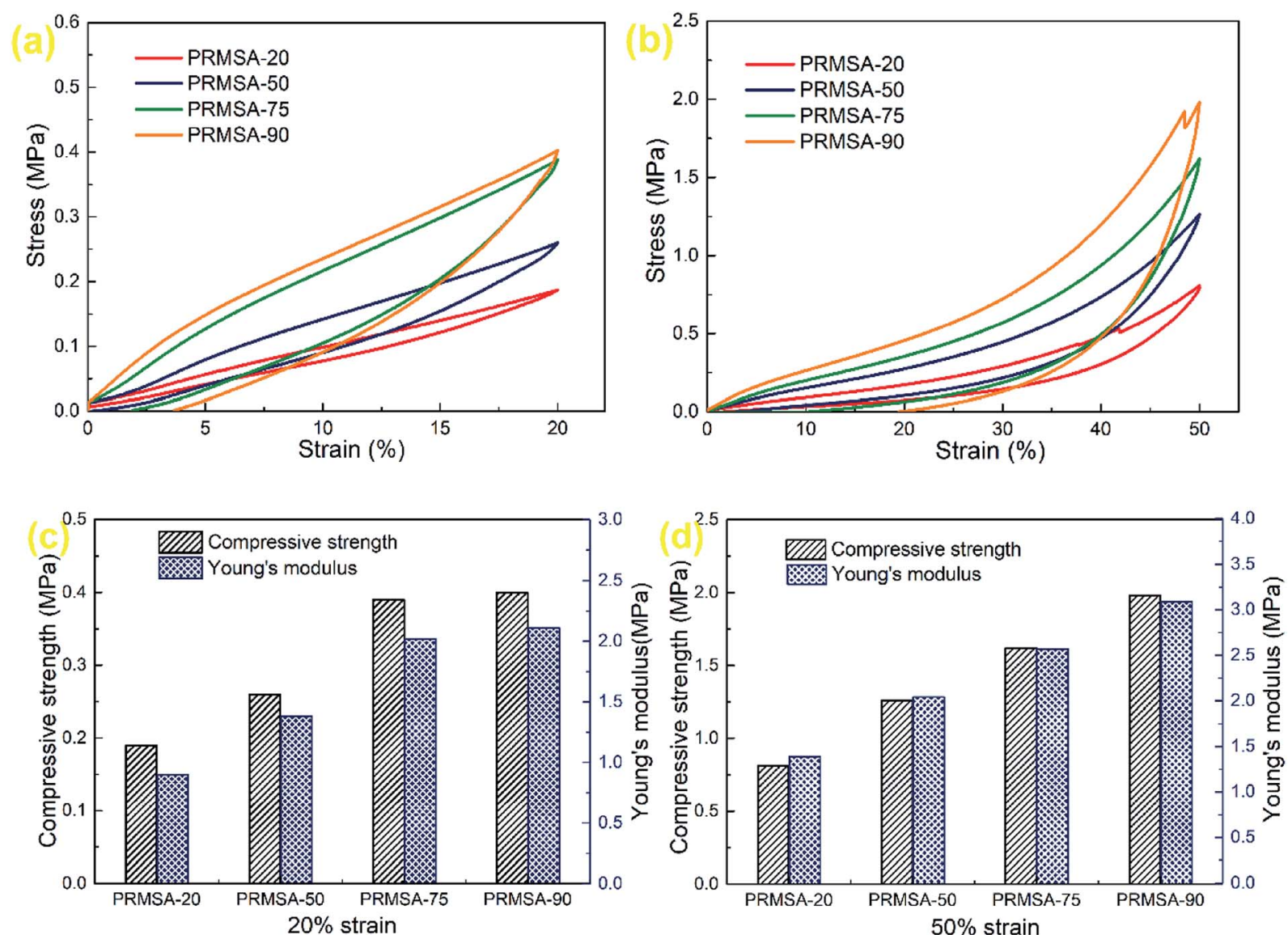

Fig. 6 Mechanical properties of PRMSAs. (a and b) Stress-strain curves of uniaxial compression-decompression tests; (c and d) compressive strengths and Young's moduli. 
components: heat transfer by the gas phase, solid phase, and radiation, ${ }^{\mathbf{3 6 - 3 8}}$ which is mainly affected by the porous structure and density. Fig. 7a displays the thermal conductivity of MSAs and PRMSAs. Affected by the non-homogeneous silica networks (Fig. 4a and b), MSA with a low density of $0.090 \mathrm{~g} \mathrm{~cm}^{-3}$ shows a relatively high thermal conductivity of $0.0620 \mathrm{~W} \mathrm{~m}^{-1} \mathrm{~K}^{-1}, 39$ the thermal conductivity of which is higher than that of PRMSAs. Interestingly, the similar finding of PRMSAs is that there is also a positive correlation relationship between low thermal conductivity and high density. For instance, PRMSA-75 with a higher density $\left(0.165 \mathrm{~g} \mathrm{~cm}^{-3}\right)$ has a relatively low thermal conductivity of $0.0228 \mathrm{~W} \mathrm{~m}^{-1} \mathrm{~K}^{-1}$ under ambient environments. This phenomenon could be explained that uniform nanopores $(\sim 11 \mathrm{~nm})$ in silica networks effectively suppress the heat transfer in PRMSA-75. Meanwhile, these results obtained from Fig. 7a also suggest that the gaseous conductivity in PRMSA-75 is much larger than that in PRMSA-20, PRMSA-50, and PRMSA-90. Owing to the macropores (larger than $50 \mathrm{~nm}$ )
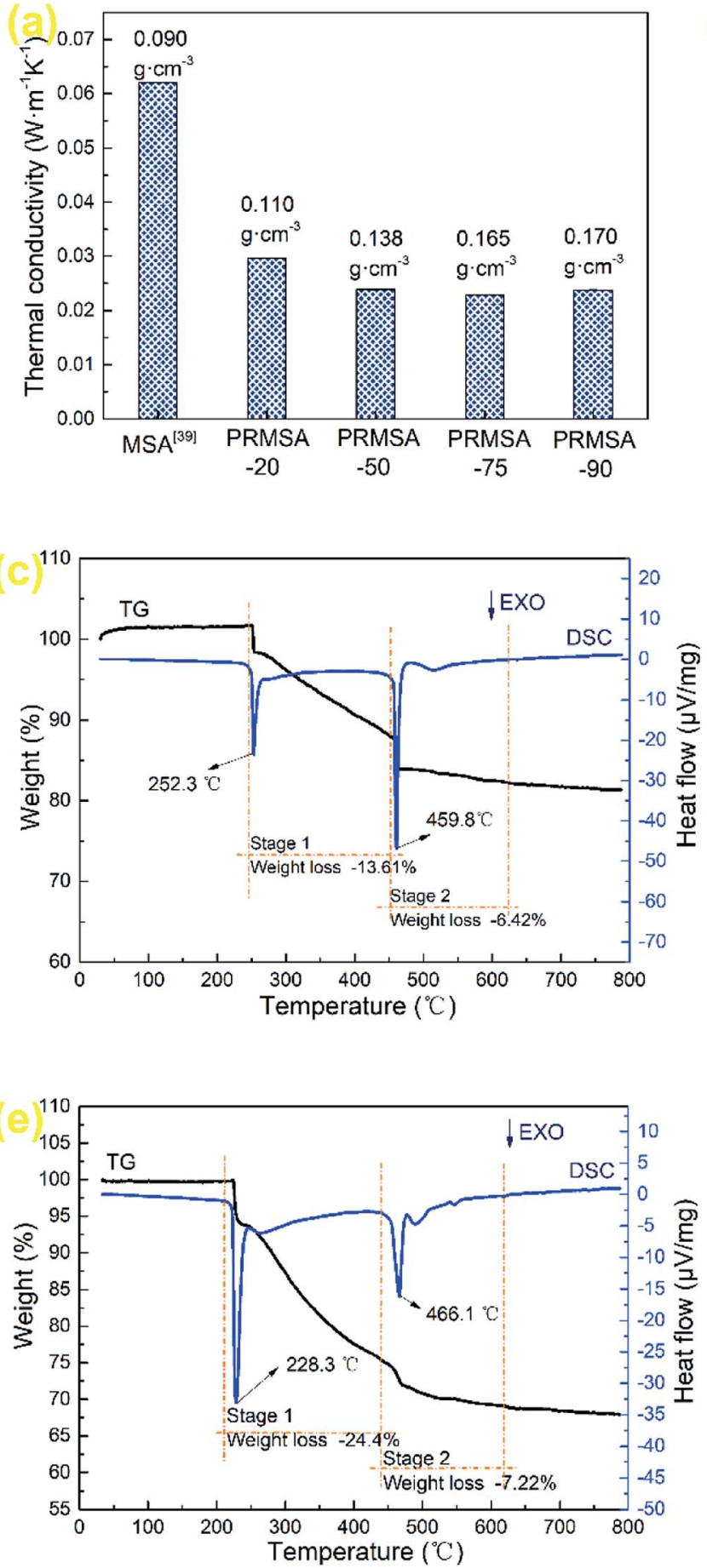
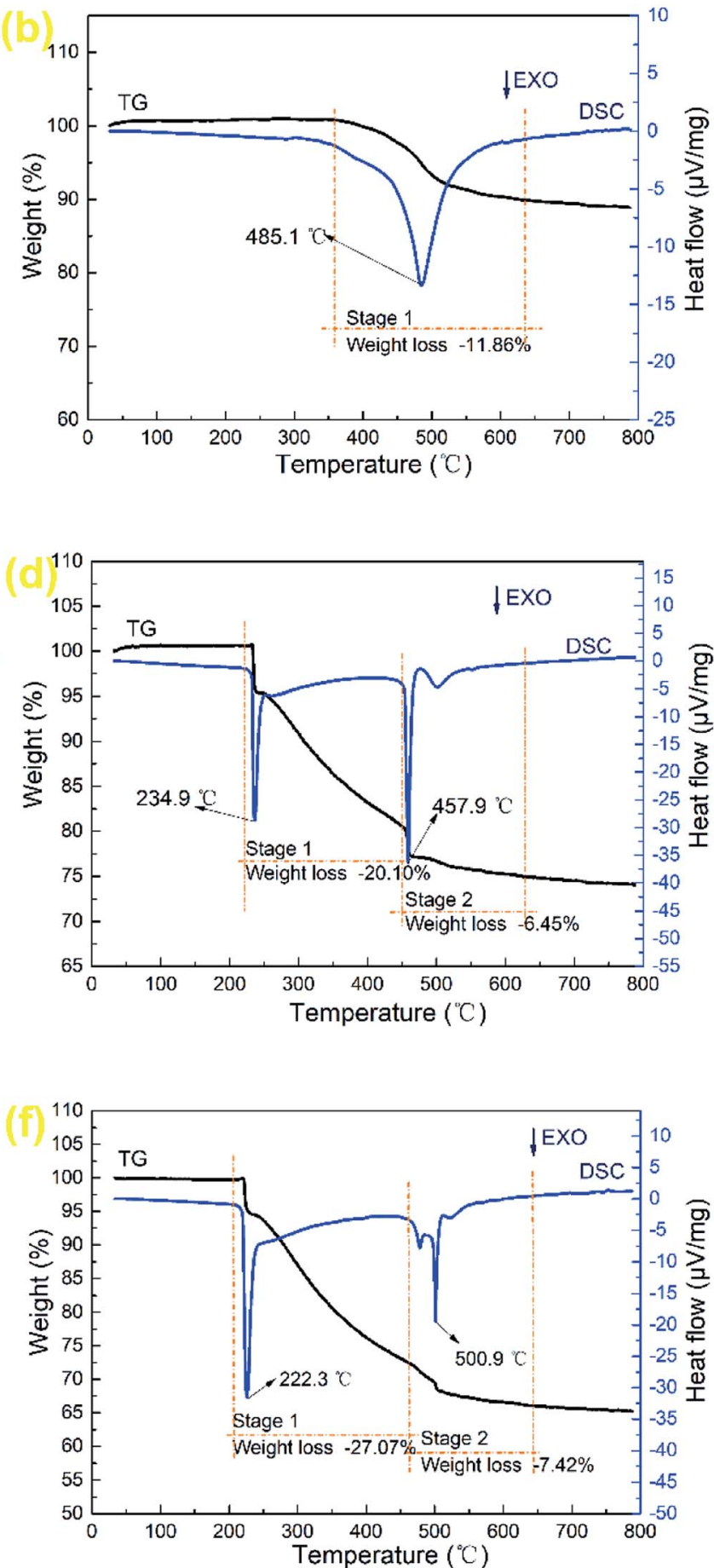

Fig. 7 Thermal properties of MSAs and PRMSAs. (a) Thermal conductivity of MSAs and PRMSAs; TG-DSC of (b) MSA; (c) PRMSA-20; (d) PRMSA50; (e) PRMSA-75; (f) PRMSA-90. 
not be detected by the $\mathrm{N}_{2}$ gas adsorption-desorption method, ${ }^{\mathbf{4 0 , 4 1}}$ the relatively higher thermal conductivity of PRMSA-20, PRMSA-50, and PRMSA-90 may be caused by some macropores in networks (Fig. 4). These macropores would lead to a high gaseous thermal conductivity in aerogel networks.

The TG-DSC curves of MSAs and PRMSAs are presented in Fig. 7b-f. As for MSAs (Fig. 7b), the predominant weight loss occurs between 350 and $650{ }^{\circ} \mathrm{C}$, owing to the thermal oxidation of $\mathrm{Si}-\mathrm{CH}_{3}$ groups on the network of MSAs. ${ }^{42}$ In this stage, the peak temperature corresponding to the exothermic reaction is 485.1 ${ }^{\circ}$ C. PRMSA-20, PRMSA-50, PRMSA-75, and PRMSA-90 have two distinct weight losses are observed in Fig. 7c-f, one of which begins at $220{ }^{\circ} \mathrm{C}$ and ends at $450{ }^{\circ} \mathrm{C}$, the other ranges from 450 to $650{ }^{\circ} \mathrm{C}$. In the first stage, the weight loss of PRMSAs is mainly caused by the thermal oxidation reactions of aliphatic hydrocarbons. Moreover, more PVMDMS polymers will result in a larger weight loss. The weight loss of PRMSA-90 is up to $27.07 \%$ before $450{ }^{\circ} \mathrm{C}$. In addition, the peak temperatures in the first stage obtained by DSC curves show similar features, which suggests that silica aerogels reinforced with PVMDMS are more suitable for applying in low temperature environments (under $200{ }^{\circ} \mathrm{C}$ ). With the increase of PVMDMS addition, the peak temperatures of exothermic reactions in the second stage are stable between 450 and $500{ }^{\circ} \mathrm{C}$, indicating that PVMDMS polymers have little effect on the thermal stability of $\mathrm{Si}-\mathrm{CH}_{3}$ groups.

\section{Conclusions}

Monolithic and elastic, polymer-reinforced silica aerogels have been synthesized by using MTMS as precursor and PVMDMS as reinforcement. The abundant methyl groups linked to silica backbone make PRMSAs exhibit high hydrophobic properties, which are fully confirmed by FTIR and TG-DSC analyses. Moreover, homogenous 3D silica networks have been observed by SEM, and the pore diameter of PRMSAs is much smaller than that of MSAs when MTMS is the sole precursor. In particular, because of the introduction of PVMDMS polymers, PRMSAs without obvious large particles possess a higher SSA, reaching up to $1039 \mathrm{~m}^{2} \mathrm{~g}^{-1}$. Compared with non-reinforced MSAs, the presence of long-chain aliphatic hydrocarbons is beneficial for PRMSAs to acquire improved mechanical properties. PRMSAs show a perfectly elastic recovery property when the critical ratio of PVMDMS is integrated into aerogel networks. As for thermal insulation, the organic components have no negative impact on thermal conductivity. On the contrary, PRMSAs exhibit a relatively low thermal conductivity of $0.0228 \mathrm{~W} \mathrm{~m}^{-1} \mathrm{~K}^{-1}$ under ambient environments, which would be of great significance to the application of thermal insulation. In conclusion, the ultimate goal of this paper is to produce polymer-reinforced silica aerogels with the desired combination of properties for a particular application. All these results would help us to have a better design of high-performance aerogel materials.

\section{Conflicts of interest}

There are no conflicts to declare.

\section{Acknowledgements}

This work was supported by National Natural Science Foundation of China (Grant no. 51712360). The authors gratefully acknowledged the Program for Technology Innovative Group of National University of Defense Technology.

\section{References}

1 N. Leventis, Three-dimensional core-shell superstructures: mechanically strong aerogels, Acc. Chem. Res., 2007, 40(9), 874-884.

2 M. J. Van Bommel and A. B. De Haan, Drying of silica aerogel with supercritical carbon dioxide, J. Non-Cryst. Solids, 1995, 186, 78-82.

3 H. Maleki, L. Durães and A. Portugal, An overview on silica aerogels synthesis and different mechanical reinforcing strategies, J. Non-Cryst. Solids, 2014, 385, 55-74.

4 D. J. Boday, P. Y. Keng, B. Muriithi, et al., Mechanically reinforced silica aerogel nanocomposites via surface initiated atom transfer radical polymerizations, J. Mater. Chem., 2010, 20(33), 6863-6865.

$5 \mathrm{M}$. Gronauer and J. Fricke, Acoustic properties of microporous $\mathrm{SiO}_{2}$-aerogel, Acta Acust. Acust., 1986, 59(3), 177-181.

6 D. D. Díaz, D. Kühbeck and R. J. Koopmans, Stimuliresponsive gels as reaction vessels and reusable catalysts, Chem. Soc. Rev., 2011, 40(1), 427-448.

7 S. Baskaran, J. Liu, K. Domansky, et al., Low dielectric constant mesoporous silica films through molecularly templated synthesis, Adv. Mater., 2000, 12(4), 291-294.

8 Y. Lin, C. Ko, C. Chen, et al., Reusable methyltrimethoxysilane-based mesoporous water-repellent silica aerogel membranes for $\mathrm{CO}_{2}$ capture, RSC Adv., 2014, 4(3), 1456-1459.

9 L. Durães, M. Ochoa, N. Rocha, et al., Effect of the drying conditions on the microstructure of silica based xerogels and aerogels, J. Nanosci. Nanotechnol., 2012, 12(8), 68286834.

10 L. Durães, A. Maia and A. Portugal, Effect of additives on the properties of silica based aerogels synthesized from methyltrimethoxysilane (MTMS), J. Supercrit. Fluids, 2015, 106, 85-92.

11 B. N. Nguyen, M. A. B. Meador, A. Medoro, et al., Elastic behavior of methyltrimethoxysilane based aerogels reinforced with tri-isocyanate, ACS Appl. Mater. Interfaces, 2010, 2(5), 1430-1443.

12 S. Hæreid, J. Anderson, M. A. Einarsrud, et al., Thermal and temporal aging of TMOS-based aerogel precursors in water, J. Non-Cryst. Solids, 1995, 185(3), 221-226.

13 S. Mulik, C. Sotiriou-Leventis, G. Churu, et al., Cross-linking 3D assemblies of nanoparticles into mechanically strong aerogels by surface-initiated free-radical polymerization, Chem. Mater., 2008, 20(15), 5035-5046.

$14 \mathrm{~J}$. Wang and X. Zhang, Binary crystallized supramolecular aerogels derived from host-guest inclusion complexes, ACS Nano, 2015, 9(11), 11389-11397. 
15 S. Zhao, Z. Zhang, G. Sèbe, et al., Multiscale assembly of superinsulating silica aerogels within silylated nanocellulosic scaffolds: improved mechanical properties promoted by nanoscale chemical compatibilization, $A d v$. Funct. Mater., 2015, 25(15), 2326-2334.

16 M. A. B. Meador, S. L. Vivod, L. McCorkle, et al., Reinforcing polymer cross-linked aerogels with carbon nanofibers, $J$. Mater. Chem., 2008, 18(16), 1843-1852.

17 H. Maleki, L. Durães and A. Portugal, Synthesis of lightweight polymer-reinforced silica aerogels with improved mechanical and thermal insulation properties for space applications, Microporous Mesoporous Mater., 2014, 197, 116-129.

18 M. A. B. Meador, A. S. Weber, A. Hindi, et al., Structureproperty relationships in porous 3D Nanostructures: epoxycross-linked silica aerogels produced using ethanol as the solvent, ACS Appl. Mater. Interfaces, 2009, 1(4), 894-906.

19 M. A. B. Meador, L. A. Capadona, L. McCorkle, et al., Structure-property relationships in porous 3D nanostructures as a function of preparation conditions: isocyanate cross-linked silica aerogels, Chem. Mater., 2007, 19(9), 2247-2260.

20 Y. Duan, S. C. Jana, B. Lama, et al., Reinforcement of silica aerogels using silane-end-capped polyurethanes, Langmuir, 2013, 29(20), 6156-6165.

21 N. Leventis, A. Sadekar, N. Chandrasekaran, et al., Click synthesis of monolithic silicon carbide aerogels from polyacrylonitrile-coated 3D silica networks, Chem. Mater., 2010, 22(9), 2790-2803.

22 B. N. Nguyen, M. A. B. Meador, M. E. Tousley, et al., Tailoring elastic properties of silica aerogels cross-linked with polystyrene, ACS Appl. Mater. Interfaces, 2009, 1(3), 621-630.

23 G. Zu, K. Kanamori, A. Maeno, et al., Superflexible multifunctional polyvinylpolydimethylsiloxane-based aerogels as efficient absorbents, thermal superinsulators, and strain sensors, Angew. Chem., Int. Ed., 2018, 57(31), 9722-9727.

24 G. Zu, T. Shimizu, K. Kanamori, et al., Transparent, superflexible doubly cross-linked polyvinylpolymethylsiloxane aerogel superinsulators via ambient pressure drying, ACS Nano, 2018, 12(1), 521-532.

25 G. Zu, K. Kanamori, T. Shimizu, et al., Versatile double-crosslinking approach to transparent, machinable, supercompressible, highly bendable aerogel thermal superinsulators, Chem. Mater., 2018, 30(8), 2759-2770.

26 R. B. Torres, J. P. Vareda, A. Lamy-Mendes, et al., Effect of different silylation agents on the properties of ambient pressure dried and supercritically dried vinyl-modified silica aerogels, J. Supercrit. Fluids, 2019, 147, 81-89.

27 A. Ayral, J. Phalippou and T. Woignier, Skeletal density of silica aerogels determined by helium pycnometry, J. Mater. Sci., 1992, 27(5), 1166-1170.
28 H. S. Yang, S. Y. Choi, S. H. Hyun, et al., Ambient-dried low dielectric $\mathrm{SiO}_{2}$ aerogel thin film, J. Non-Cryst. Solids, 1997, 221(2-3), 151-156.

29 T. Matias, C. Varino, H. C. de Sousa, et al., Novel flexible, hybrid aerogels with vinyl- and methyltrimethoxysilane in the underlying silica structure, J. Mater. Sci., 2016, 51(14), 6781-6792.

30 A. C. Pierre, E. Elaloui and G. M. Pajonk, Comparison of the structure and porous texture of alumina gels synthesized by different methods, Langmuir, 1998, 14(1), 66-73.

31 S. D. Bhagat, C. S. Oh, Y. H. Kim, et al., Methyltrimethoxysilane based monolithic silica aerogels via ambient pressure drying, Microporous Mesoporous Mater., 2007, 100(1-3), 350-355.

32 K. Kanamori, M. Aizawa, K. Nakanishi, et al., New transparent methylsilsesquioxane aerogels and xerogels with improved mechanical properties, Adv. Mater., 2007, 19(12), 1589-1593.

33 N. Leventis, C. Sotiriou-Leventis, G. Zhang, et al., Nanoengineering strong silica aerogels, Nano Lett., 2002, 2(9), 957-960.

34 F. Ilhan, E. F. Fabrizio, L. McCorkle, et al., Hydrophobic monolithic aerogels by nanocasting polystyrene on aminemodified silica, J. Mater. Chem., 2006, 16(29), 3046-3054.

35 H. Guo, M. A. B. Meador, L. McCorkle, et al., Polyimide aerogels cross-linked through amine functionalized polyoligomeric silsesquioxane, ACS Appl. Mater. Interfaces, 2011, 3(2), 546-552.

36 X. Lu, O. Nilsson, J. Fricke, et al., Thermal and electrical conductivity of monolithic carbon aerogels, J. Appl. Phys., 1993, 73(2), 581-584.

37 X. Lu, P. Wang, M. C. Arduini-Schuster, et al., Thermal transport in organic and opacified silica monolithic aerogels, J. Non-Cryst. Solids, 1992, 145(145), 207-210.

38 X. Lu, M. C. Arduini-Schuster, J. Kuhn, et al., Thermal conductivity of monolithic organic aerogels, Science, 1992, 255(5047), 971-972.

39 D. Y. Nadargi and A. V. Rao, Methyltriethoxysilane: new precursor for synthesizing silica aerogels, J. Alloys Compd., 2009, 467(1-2), 397-404.

40 G. Reichenauer and G. W. Scherer, Nitrogen sorption in aerogels, J. Non-Cryst. Solids, 2001, 285(1-3), 167-174.

41 G. Reichenauer and G. W. Scherer, Effects upon nitrogen sorption analysis in aerogels, J. Colloid Interface Sci., 2001, 236(2), 385-386.

42 Z. Li, X. Cheng, S. He, et al., Characteristics of ambientpressure-dried aerogels synthesized via different surface modification methods, J. Sol-Gel Sci. Technol., 2015, 76(1), 138-149. 